ARTIGO DE REVISÃO

ISSN 1677-5090

(C) 2019 Revista de Ciências Médicas e Biológicas

\title{
Fatores de risco da síndrome da apneia obstrutiva do sono no adulto
}

\author{
Risk factors for obstructive sleep apnea syndrome in adults
}

\begin{abstract}
Thomas Peter Maahs ${ }^{1}$, Marcia Angelica Peter Maahs²*, Gerson Schulz Maahs ${ }^{3}$
${ }^{1}$ Graduando em Medicina da Universidade Federal de Ciências da Saúde de Porto Alegre (UFCSPA); ${ }^{2}$ Doutora em Odontologia pela Pontifícia Universidade Católica do Rio Grande do Sul (PUCRS), Pós-doutora em Patologia pela UFCSPA, Professora Adjunta de Odontologia e Ortodontia do Departamento de Fonoaudiologia e do Programa de Pós-graduação em Ciências da Reabilitação (PPGCR) UFCSPA; ${ }^{3}$ Mestrado e Doutorado em Medicina e Ciências da Saúde pela PUCRS, Professor Adjunto de Otorrinolaringologia subárea de Cirurgia de Cabeça e Pescoço da Faculdade de Medicina da Universidade Federal do Rio Grande do Sul (UFRGS).
\end{abstract}

\begin{abstract}
Resumo
Introdução: a síndrome da apneia obstrutiva do sono (SAOS) caracteriza-se por episódios recorrentes de obstrução parcial ou total da via aérea superior, normalmente acompanhada por roncos. É de etiologia multifatorial, e o diagnóstico leva em consideração os fatores de risco e as comorbidades. Objetivo: estudar os fatores de risco da SAOS, devido às comorbidades e às consequências graves que podem estar relacionadas à patologia, visando a atuar em sua prevenção e tratamento. Revisão de literatura: os principais fatores de risco encontrados foram: obesidade, circunferência cervical maior que $40 \mathrm{~cm}, I \mathrm{IMC}$ aumentado, histórico familiar, sexo masculino, meia-idade, Classe III e IV de Mallampati modificada e alterações nas vias aéreas superiores como desvio de septo nasal, palato mole rebaixado e posteriorizado e alterações craniofaciais. Metodologia: realizou-se uma revisão de literatura sobre os fatores de risco da SAOS, utilizando-se as bases de dados Pubmed, Scielo e Medline e os descritores de busca de acordo com os DECS ou Mesh. Conclusão: o estudo dos fatores de risco associados à presença de SAOS demonstra a necessidade da abordagem interdisciplinar dessa patologia junto ao médico otorrinolaringologista, por ser ela multifatorial.

Palavras-chave: Apneia Obstrutiva do Sono. Fatores de risco. Síndrome da Apneia do Sono.
\end{abstract}

\begin{abstract}
Introduction: obstructive sleep apnea syndrome (OSAS) is characterized by recurrent episodes of partial or total obstruction of the upper airways, usually accompanied by snoring. It has a multifactorial etiology, and the diagnosis takes into account risk factors and comorbidities. Objective: to study the risk factors for OSAS, with regard to morbidities and the serious consequences that may be related to its pathology, aiming at its prevention and treatment. Literature review: according to the literature, the main risk factors are: obesity, cervical circumference greater than $40 \mathrm{~cm}$, increased BMI, family history, male gender, middle age, modified Mallampati Class III o IV, and alterations in the upper airways such as deviated nasal septum and lowered and posterior soft palate and craniofacial changes. Methodology: a review of the literature on the risk factors for OSAS was carried out using the PubMed, Scielo and Medline databases and the search descriptors according to the DECS or Mesh. Conclusion: a study of the risk factors associated with OSAS demonstrates the need for an interdisciplinary approach to this pathology with the otorhinolaryngologist, because of the multifactorial nature of OSAS.
\end{abstract}

Keywords: Obstructive sleep apnea. Risk factors. Obstructive sleep apnea syndrome.

\section{INTRODUÇÃO}

A síndrome da apneia obstrutiva do sono (SAOS) caracteriza-se por episódios recorrentes de obstrução parcial ou total da via aérea superior, que envolvem cessação ou diminuição significativa do fluxo aéreo, com hipoxemia intermitente, despertares frequentes do sono e dessaturações de oxihemoglobina recorrentes, o que interfere nos padrões normais de sono, gerando dificuldade de adormecer, sono irregular e ronco ${ }^{1}$. Os sinais de alerta

Correspondente/Corresponding: Correspondente/Corresponding: *Marcia Angelica Peter Maahs - End: Av. Sarmento Leite, 245 - Porto Alegre, Rio Grande do Sul. CEP: 90050-170 - Tel: (51) 3303-9000 -E-mail: marciama@ufcspa.edu.br mais frequentes para a síndrome são roncos noturnos, sonolência diurna excessiva e pausas respiratórias presenciadas por outra pessoa durante o sono ${ }^{2}$.

A prevalência da SAOS em adultos é $4 \%$ nos homens e $2 \%$ nas mulheres de meia-idade ${ }^{3}$. Porém as mulheres, após a menopausa, passam a ter o mesmo risco dos homens para desenvolver a doença ${ }^{4}$.

A SAOS possui herdabilidade ${ }^{5}$. Sua etiologia multifatorial é decorrente, em parte, de alterações anatômicas da via aérea superior e do esqueleto craniofacial, associadas a alterações neuromusculares da faringe e obesidade ${ }^{6}$.

O diagnóstico envolve anamnese, exame físico e exames complementares, levando-se em consideração os fatores de risco e as comorbidades. Os principais fatores de risco da SAOS são: sexo masculino, obesidade, características crânio e orofaciais, como retromicrognatia, 
atresia maxilar transversa e língua retroposicionada ${ }^{4,7}$, 8 , e as comorbidades mais comuns são anormalidades cardiovasculares e metabólicas ${ }^{1,9}$. Dentre as anormalidades cardiovasculares, encontram-se hipertensão, doença arterial coronariana, arritmias cardíacas, morte súbita cardíaca e insuficiência cardíaca9 ${ }^{9}$. Além disso, a SAOS pode contribuir para o acidente vascular cerebral (AVC) isquêmico, não apenas como um fator predisponente, mas também como um fator desencadeante. $O$ tratamento da SAOS pode, portanto, ser benéfico na prevenção do AVC, particularmente no que ocorre durante o sono ${ }^{10}$.

Quanto aos exames complementares de diagnóstico, a análise cefalométrica pode ser utilizada, pois ela pode quantificar o estreitamento do espaço aéreo faríngeo (EAF) que contribui para SAOS. Além disso, ela possibilita verificar o padrão esquelético, uma vez que a maxila e a mandíbula retrognatas, comuns nesses pacientes, contribuem para o quadro . No entanto, a polissonografia é o exame "padrão ouro" para o diagnóstico da SAOS, e sua interpretação permite, por meio do índice de apneia hipopneia (IAH), classificá-la em: apneia leve, de 5 a 15; moderada de 15 a 30; e grave, acima de 30 eventos por hora de sono. Abaixo de 5, considera-se normal².

Para o tratamento da SAOS, a perda de peso é geralmente recomendada como tratamento primordial, em pessoas obesas com a patologia. Os demais métodos de tratamento, clínicos e (ou) cirúrgicos, levam em consideração os fatores anatômicos causadores, a gravidade da doença, as comorbidades e a adesão ao tratamento ${ }^{12}$.

Em função das comorbidades e consequências graves que podem estar relacionadas à SAOS, torna-se importante o estudo dos fatores de risco dessa patologia, no intuito de poder atuar em sua prevenção e tratamento.

\section{REVISÃO DE LITERATURA}

Dentre os fatores de risco da SAOS em adultos, encontrados na literatura, destacam-se obesidade, circunferência cervical maior que $40 \mathrm{~cm}$, IMC (índice de massa corporal) aumentado, histórico familiar, sexo masculino, meia-idade, Classe III e IV da classificação de Mallampati modificada, baixa atividade física e alterações nas vias aéreas superiores, como o desvio de septo nasal, a macroglossia, as alterações craniofaciais, a abóboda palatina alta, as alterações de forma e tamanho do palato mole e o osso hioide baixo ${ }^{4,7,8,13-16}$.

A obesidade é o maior fator de risco para a SAOS: em torno de $70 \%$ dos pacientes com SAOS são obesos. Esses pacientes apresentam risco significativo de obstrução das vias aéreas superiores, pois os tecidos faríngeos têm maior deposição de gordura, causando excesso de tecido nas vias aéreas superiores e um aumento da probabilidade de colapso da parede da faringe, resultando em obstrução das vias aéreas. Esse quadro pode ser piorado quando pacientes obesos recebem medicamentos que deprimem o sistema nervoso central ${ }^{14}$.
A classificação de Mallampati modificada expressa a proporção dos tecidos moles da cavidade bucal com a orofaringe. Avalia-se o paciente sentado, com a boca em abertura máxima e a língua relaxada, visando a observar a dimensão de exposição da orofaringe. A classificação vai de I a IV, de acordo com a visualização de mais a menos do bordo livre do palato mole em relação à base da língua. Na Classe I, palato mole, fauce, úvula e pilares amigdalianos são visíveis; na Classe II, palato mole, fauce e úvula são visíveis; na Classe III, o palato mole e a base da úvula são visíveis; e na Classe IV, o palato mole é totalmente não visível ${ }^{17}$.

Alterações crâniofaciais que levam ao estreitamento do espaço aéreo faríngeo são fatores de risco comumente relacionados a SAOS. Existe forte evidência de redução do espaço aéreo faríngeo quando o osso hioide se situa em posição inferior em pacientes adultos com SAOS. Quanto maior a distância do osso hioide, no plano mandibular, maior a severidade da $\mathrm{SAOS}^{18}$, e indivíduos com face longa têm as vias aéreas superiores mais estreitas. Os portadores de retrognatismo mandibular têm a orofaringe e a hipofaringe estreitadas, o que pode contribuir para existência da SAOS, e o retrognatismo maxilar contribui para o estreitamento da nasofaringe $\mathrm{e}^{8,11,12}$. O tratamento ortocirúrgico pode levar ao aumento do espaço aéreo faríngeo (Figura 1$)^{19}$ e contribuir para o tratamento de pacientes com SAOS ${ }^{12}$.

Figura 1 - Telerradiografia de perfil do espaço aéreo faríngeo no pré-cirúrgico $(A)$ e no pós-cirúrgico $(B)$ de tratamento ortocirúrgico para Classe ll esquelética por avanço maxilomandibular.
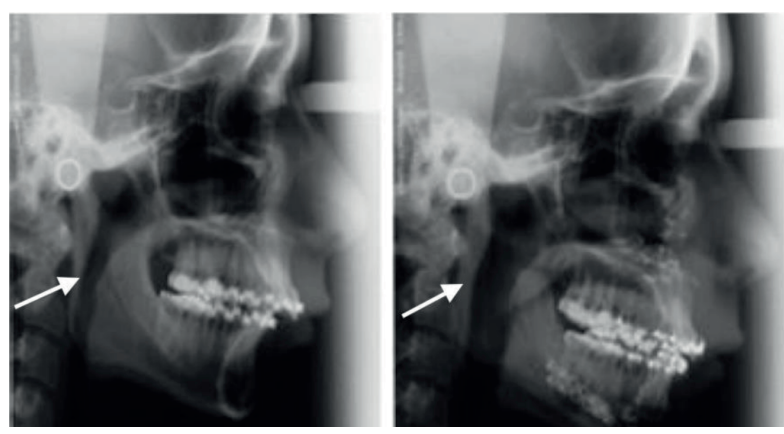

Fonte: Adaptada de Maahs, Ferreira e Puricelli ${ }^{19}$

Além do retrognatismo de maxila e (ou) de mandíbula, dentre as características crânio-esqueléticas que podem contribuir para a presença da SAOS, por levarem à diminuição do espaço aéreo faríngeo ${ }^{11}$, encontra-se a atresia maxilar transversa, que leva a uma postura baixa e posteriorizada da língua, diminuindo o espaço retroglossal ${ }^{8}$.

\section{METODOLOGIA}

Esta revisão de literatura utilizou referências publicadas em âmbito nacional e internacional. As bases de 
dados utilizadas foram: Pubmed, Scielo (Scientific Electronic Library Online) e Medline (Medical Literature Analysis and Retrieval System Online), por incluírem a maior parte das publicações sobre o tema. Como descritores de busca, foram utilizados os seguintes termos, de acordo com os "Descritores em Ciências da Saúde" (DECs) ou Medical Subject Headings (Mesh): apneia obstrutiva do sono, fatores de risco, síndrome da apneia obstrutiva do sono e seus respectivos termos em língua inglesa (obstructive sleep apnea, risk factors, obstructive sleep apnea syndrome), bem como a combinação entre si com os operadores booleanos AND ou OR. Os critérios de inclusão foram publicações que abordassem os descritores de busca utilizados. Os dados foram analisados por meio da leitura dos resumos utilizados na seleção do tema. Os artigos descritos nas referências foram lidos para realização da descrição e discussão sobre os principais fatores de risco da SAOS.

\section{DISCUSSÃO}

\section{Obesidade, circunferência cervical aumentada e IMC aumentado}

Apesar da frequente relação com a obesidade, nem todo paciente com SAOS é obeso ${ }^{13}$, embora cerca de $2 / 3$ o sejam ${ }^{16}$.

Existe uma tendência de os pacientes de meia-idade com SAOS serem mais propensos à obesidade do que os pacientes mais jovens ou mais velhos com SAOS. Em particular, o valor preditivo de circunferência cervical é significativamente menor para pacientes com SAOS mais jovens e mais velhos. Além disso, a circunferência cervical aumentada tem relação com a severidade da SAOS em pacientes entre 40 e 60 anos de idade ${ }^{18}$, e a circunferência cervical maior que $40 \mathrm{~cm}$ é considerada um fator independente de alto risco a SAOS ${ }^{13}$.

O índice de massa corporal (IMC) aumentado é um indicativo de sobrepeso e tem relação direta com a prevalência da SAOS, sendo os indivíduos com IMC acima de $30 \mathrm{Kg} / \mathrm{m}^{2}$ mais propensos a essa patologia. ${ }^{20}$

\section{Histórico familiar}

Existe risco aumentado de SAOS em uma família em que um membro é afetado por essa enfermidade. A prevalência da SAOS entre parentes de primeiro grau tem uma variação entre 22 a $84 \%{ }^{5}$. Essa incidência familiar pode ser explicada devido ao fato de a maioria dos fatores de risco para desenvolver a doença serem determinados geneticamente, como a obesidade e as características craniofaciais $^{21}$.

\section{Sexo masculino}

Homens são mais acometidos que mulheres, provavelmente por fatores anatômicos relacionados ao sexo ${ }^{16}$, como a tendência a depositar mais gordura na região cervical.

\section{Idade}

O pico de incidência situa-se entre os 40 e 50 anos, e as mulheres com SAOS frequentemente se encontram no climatério, o que sugere uma influência hormonal na fisiopatologia do colabamento das vias aéreas durante o sono ${ }^{16}$.

\section{Classe III e IV de Mallampati}

Classe III e IV de Mallampati também constituem fatores independentes de alto risco para a SAOS. A Classe III de Mallampati ocorre quando, em exame oral, apenas o palato mole e a base da úvula são visíveis; e a Classe IV, quando o palato mole é totalmente não visível ${ }^{13}$.

\section{Baixa atividade física}

A baixa atividade física tem sido apontada como fator de risco para a SAOS. Inclusive sugere-se que a redução de peso, juntamente com uma dieta saudável e aumento da atividade física podem corrigir ou, pelo menos, melhorar os sintomas da SAOS 22 .

\section{Alterações nas vias aéreas superiores}

Dentre as anormalidades anatômicas faciais mais relacionadas com a obstrução da via aérea superior em adultos e predisponentes de SAOS, está o desvio de septo nasal ${ }^{15}$, o palato mole rebaixado e posteriorizado, e as alterações craniofaciais. Também há relatos de que quanto maior o comprimento do palato mole, maior a severidade da $\mathrm{SAOS}^{18}$.

\section{CONCLUSÃO}

O estudo dos fatores de risco associados à presença da SAOS demonstra seu caráter multifatorial, o que demanda uma abordagem interdisciplinar dessa patologia junto ao médico otorrinolaringologista.

\section{REFERÊNCIAS}

1. CASTANEDA, A. et al. Correlation between metabolic syndrome and sleep apnea. World. J. Diabetes., Pleasanton, v.9, n.4, p.66-71, 2018. DOI: 10.4239/wjd.v9.i4.66.

2. BERRY, R.B. et al. AASM scoring manual version 2.2 updates: New chapters for scoring infant sleep staging and home sleep apnea testing. J. Clin. Sleep Med., Seatle, v.11, n.11, p.1253-1254, 2015. DOI: 10.5664/ jcsm.5176.

3.YOUNG, T. et al. The occurrence of sleep-disordered breathing among middle-aged adults. N. Engl. J. Med., v.328, n.17, p.1230-1235, Apr.1993.

4. ZANCANELLA, E. et al. Obstructive sleep apnea and primary snoring: diagnosis. Braz. J. Otorhinolaryngol., São Paulo, v. 80, n.1 Suppl 1:S1-16, Jan.-Fev. 2014. DOI: 10.5935/1808-8694.2014S001.

5.MARTINS, A. B.; TUFIK, S.; MOURA, S. M. Physiopatology of obstructive sleep apnea-hypopnea syndrome. J. Bras. Pneumol. Brasília, v.33, n.1, p. 93-100.e, Jan./ Fev. 2007.

6. AMERICAN ACADEMY OF SLEEP MEDICINE TASK FORCE. Sleep-related breathing disorders in adults: recommendations for sybdrome definition 
and measurement techniques in clinical research. Sleep, Oxford, n.22, p.667-689, Aug. 1999.

7. LOWE, A. A.; SANTAMARIA, J. D.; FLEETHAM, J. A. Facial morphology and obstructive sleep apnea. Am. J. Orthod. Dentofacial. Orthop., St. Louis, v.90, v.6, p.484-491, 1986.

8. CISTULLI, P. A.; PALMISANO, R. G.; POOLE, M. D. Treatment of obstructive sleep apnea syndrome by rapid maxillary expansion. Sleep., Oxford, v.21, n.8, p.831-835,1998.

9. KHATTAK, H. K. et al. Obstructive Sleep Apnea in Heart Failure: Review of Prevalence, Treatment with Continuous Positive Airway Pressure, and Prognosis. Tex Heart Inst J., Houston, v.45, n.3, p.151-161, Jun. 2018. DOI: 10.14503/THIJ-15-5678.

10. KIM, J. S. et al. Increased Risk of Ischemic Stroke during Sleep in Apneic Patients. J. Clin. Neurol., [s.I], v.14, n.2, p.174-8, 2018. DOI: 10.3988/jcn.2018.14.2.174.

11. LOWE, A. A.; SANTAMARIA, J. D.; FLEETHAM, J. A. Facial morphology and obstructive sleep apnea. Am. J. Orthod.Dentofacial. Orthop., St. Louis, v. 90, n.6, p. 484-491, 1986.

12. MAAHS, M. A. P.; ALMEIDA, S. T. Respiração oral e apneia obstrutiva do sono: integração no diagnóstico e tratamento. Rio de Janeiro, Editora Revinter. 2017. 456 p.

13. KALE, S. S.; KAKODKAR, P.; SHETIYA, S. H. Assessment of oral findings of dental patients who screen high and no risk for obstructive sleep apnea (OSA) reporting to a dental college - A cross sectional study. Sleep Sci., São Paulo, v.11, n.2, p.112-117, Mar./ Apr. 2018. DOI: 10.5935/1984-0063.20180021.

14. CASTRO, D.; FReEMAN, L. A. Airway, Oropharyngeal. Stat Pearls Publishing, [s.I], Oct. 2017.
15. MELLO JUNIOR, C. F. Radiological findings in patients with obstructive sleep apnea. J. Bras. Penumol., Brasília, v.39, n.1, p.98-101. Jan./Fev. 2013. DOI: $10.1590 /$ S1806-37132013000100014.

16.WIEGAND, L.; ZWILICH, C. W. Obstructive Sleep Apnea. In Bone $R C$ (Ed) Disease-a-Month. St. Louis, Mosby Year Book, v.XL, n.4, p.199252, 1994.

17. SAMSOON, G.L.; YOUNG, J.R. Difficult tracheal intubation: a retrospective study. Anaesthesia. v.42, p. 487-490, 1987.

18. BORGES, P. T. M. Medidas cefalométricas e antropométricas do índice de apneia e hipopneia em diferentes faixas etárias. Braz. J. Otorhinolaryngol., São Paulo, v.80, n.1, p.79-84. 2015. DOI:10.1016/j. bjorl.2014.06.001.

19. MAAHS, M. A. P.; FERREIRA, E. S.; PURICELLI, E. Estabilidade da dimensão do espaço aéreo faríngeo após o avanço maxilomandibular em pacientes com maloclusão de classe II. RFO UPF., Passo Fundo, v. 16, n.2, p. 154-160, Mai-Ago. 2011.

20. WORLD HEALTH ORGANIZATION. Obesity: Preventing and managing the global epidemic. Report of a WHO consultation. World Health Organ Tech Rep. Ser., Geneva, v.894, n.i-xiii, p.1-253, 2000.

21. PETRUCO, A. C. M; BAGNATO, M.C. Aspectos genéticos da SAOS. J. Bras. Pneumol., Brasília, v.36, n.2, p.S1-S61, 2010.

22. TUOMILETRO, H.; SEPPA, J.; UUSITUPA, M. Obesity and obstructive sleep apnea--clinical significance of weight loss. Sleep. Med. Rev., v.17, n.5, p.321-329, 2013. DOI: 10.1016/j.smrv.2012.08.002.

Submetido em: $11 / 08 / 2018$

Aceito em: 05/09/2019 